\title{
LOGARITHMS OF MATRICES
}

\author{
BURRELL W. HELTON
}

1. Introduction. This paper contains proofs of several theorems concerning logarithms of matrices such as $\log A$ exists if $A^{-1}$ exists, the set of logarithms of $I$ is uncountable, each commutative subset of the logarithms of a matrix $A$ is countable, and each commutative subset of the logarithms of $I$ is a finite-dimensional vector space over the set of integers. Most of these proofs also hold for Banach algebras with suitable norms.

2. Definitions. $M$ denotes the algebra of $n \times n$ matrices of complex numbers and $|\cdot|$ denotes a norm with respect to which $M$ is complete, $|I|=1$, and $|A|=0$ if and only if $A=0$. Capital letters will be used to represent elements of $M$, bold-faced letters for sets, and lower case letters for real numbers. Reduced fraction means a rational number expressed as a reduced fraction. The following definitions will be used.

Definition 1. $\operatorname{Exp} A=E(A)=\sum_{n=0}^{\infty}(1 / n !) A^{n}$ and $A$ is a logarithm of $B$ if and only if $B=E(A)$.

Definition 2. $\log A$ denotes the subset of $M$ such that $B \in \log A$ if and only if $B$ is a logarithm of $A$.

Definition 3. $A$ is nonsingular means $A^{-1}$ exists.

Definition 4. $A$ is a reduced logarithm of $I$ means $A \in \log I$ and if $0<|p|<1$, then $p A \notin \log I$.

Definition 5. $B$ is a preferred logarithm of $A$ means $B \in \log A$ and if $A$ commutes with $C$, then $B$ commutes with $C$.

3. Theorems. A proof of Theorem 1 can be found in [1, p. 167].

Theorem 1. If $M=E(A), N=E(B)$ and $A B=B A$, then

$$
E(A) E(B)=E(A+B)
$$

and

$$
A+B \in \log M N .
$$

THEOREM 2. If $B$ is a continuous function of bounded variation from $[0,1]$ into $M$ such that $B^{-1}$ exists, all values of $B$ commute and $B(0)=1$, then $\int_{0}^{x} B^{-1} d B$ is a preferred logarithm of $B(x)$ for $0 \leqq x \leqq 1$.

OUTLINE OF PROOF.

Received by the editors February 7, 1967. 


$$
\begin{aligned}
B(x)=1 & +\int_{0}^{x} B B^{-1} d B=1+\int_{0}^{x} B^{-1}(t) d B(t) \\
& +\int_{0}^{x}\left[\int_{0}^{t} B^{-1}(p) d B(p)\right] B^{-1}(t) d B(t)+\cdots \\
= & \sum_{n=0}^{\infty}(1 / n !)\left(\int_{0}^{x} B^{-1} d B\right)^{n} .
\end{aligned}
$$

The following theorem, due to M. Nagumo [4, p. 67], follows as a corollary to the preceding theorem.

Theorem 3. If $A^{-1}$ exists, then $A$ has a preferred logarithm.

Proof. Since there are only a finite set of values $z$ for which $[I+z(A-I)]^{-1}$ does not exist and since $A^{-1}$ exists, there is a continuous function $g$ of bounded variation from $[0,1]$ to the complex numbers such that $g(0)=0, g(1)=1$ and, if $B(x)=[I+g(x)(A-I)]$, then $B$ satisfies the hypothesis of Theorem 2. Since $B(1)=A$, then $\int_{0}^{1} B^{-1} d B \in \log B(1)=\log A$. The referee has pointed out that the argument used in Theorems 2 and 3 yields the more general Theorem 9.5.1 in Hille and Phillips [3, p. 285].

Theorem 4. If $A \in \log I$ and $|A|<1$, then $A=0$.

Proof. $0=E(A)-I=\left(I+\sum_{k=1}^{\infty} A^{k} / k !\right)-I=A(I+B)$, where $B$ $=A / 2 !+A^{2} / 3 !+\cdots$. Since $|B|<1$, then $(I+B)^{-1}$ exists and $A=0$.

TheOREM 5. Log $I$ is an uncountable set.

Proof. There exists uncountably many pairs $a, b$ of positive numbers such that $a^{2}+b^{2}=1$ and such that

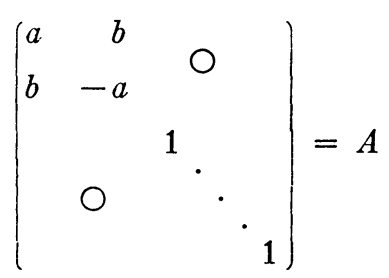

is a nonsingular square root of $I$. Hence, there exist uncountably many matrices of the form $\log A$ such that $I=E(2 \log A)$.

Theorem 6. If $\boldsymbol{S}$ is a commutative subset of $\log A$, then $\boldsymbol{S}$ is countable.

Proof. If $S$ is an uncountable subset of $\log A$, then $S$ has an 
uncountable bounded subset which has an accumulation point. Hence, there exist matrices $B, C \in S$ such that $0<|B-C|<1$ and $E(B)=A=E(C)$. Therefore, $E(B-C)=I$ and by Theorem $4 B-C=0$ which contradicts $0<|B-C|$.

Theorem 7. If $0 \neq A \in \log I$, then there is a unique positive number $n$ such that if $k$ is a real number, then $k / n A \in \log I$ if and only if $k$ is an integer. Furthermore, $n$ is an integer.

Proof. Suppose $0 \neq A \in \log I$ and $S$ is the set of positive numbers such that $h \in S$ if and only if $h A \in \log I$. The set $S$ has a greatest lower bound $m$; furthermore, $m \in S$. If $m \notin S$, there are numbers $p, q \in S$ such that $0<|(p-q)|<1$ and by Theorem $4(p-q) A=0$; this last equation contradicts the preceding inequality.

If $k$ is an integer, then $k m A \in \log I$ because $m A \in \log I$. If $k$ is a real number such that $k m A \in \log I$, then $k$ is an integer; if this is false, then there is an integer $h$ such that $1>h-k>0,(h-k) m A$ $\in \log I$, and $m>(h-k) m \in S$. Since $(1 / m) m A=A \in \log I$, then $1 / m$ is a positive integer $n$ such that $m A=(1 / n) A$. Furthermore, $n$ is unique, because if $p$ is a number satisfying these conditions, then $((p+n) / p)((1 / n) A)=((p+n) / n)((1 / p) A)=(1 / n+1 / p) A \in \log I$, $(p+n) / p$ and $(p+n) / n$ are integers, $n$ divides $p$ and $p$ divides $n$, and $n=p$.

THEOREM 8. If $A$ and $B$ commute and are reduced logarithms of $I$ and $a / p$ and $b / q$ are reduced fractions such that $(a / p) A+(b / q) B \in \log I$, then $p=q$.

Proof. $(a / p) A+(b / q) B \in \log I \rightarrow a A+(b p / q) B \in \log I \rightarrow(b p / q) B$ $\in \log I \rightarrow q$ divides $b p \rightarrow q$ divides $p$. Similarly, $p$ divides $q$; hence, $p=q$.

Theorem 9. If $B$ is a preferred logarithm of $A$ and $A^{1 / m}$ is an mth root of $A$ such that $\left(A^{1 / m}\right)^{-1}$ exists, then there is an mth root $I^{1 / m}$ of $I$ such that $A^{1 / m}=E(B / m) I^{1 / m}$.

Proof. Since $\left(A^{1 / m}\right)^{-1}$ exists, $A^{1 / m}$ has a preferred logarithm $C$. Furthermore, $B$ and $C$ commute because $A$ and $A^{1 / m}$ commute. Since $E(m C)=A=E(B)$, then $E(m C-B)=I$ and there is an $m$ th root $I^{1 / m}$ of $I$ such that $E(C-B / m)=I^{1 / m}$. Hence,

$$
E(B / m) I^{1 / m}=E(B / m) E(C-B / m)=E(C)=A^{1 / m} .
$$

LEMMA 1. If $b$ is an irrational number and $a>0$, there are integers $p$ 
and $q$ and an irrational number $r$ such that $p b=q+r$ and $|r|<a$ (Corollary to $[2$, Theorem $36, p .30])$.

THEOREM 10. If $\left\{A_{i}\right\}_{i=0}^{m}$ is a commutative sequence of linearly independent logarithms of $I$ and $\left\{a_{i}\right\}_{i=0}^{m}$ is a sequence of real numbers such that $\sum_{i=0}^{m} a_{i} A_{i} \in \log I$, then $a_{0}$ is a rational number.

Proof. Suppose $\left\{A_{i}\right\}_{i=0}^{m}$ and $\left\{a_{i}\right\}_{i=0}^{m}$ satisfy the hypothesis and that $a_{0}$ is an irrational number. From the lemma, if $1>c>0$, there exist integers $p$ and $q$ and an irrational number $b_{0}$ such that $p a_{0}$ $=q+b_{0}$ and $\left|b_{0}\right|<c$. Furthermore, $\sum_{i=0}^{m} p a_{i} A_{i} \in \log I$. Let $\left\{b_{i}\right\}_{i=1}^{m}$ be the sequence of numbers such that for $i=1, \cdots, m, b_{i}=p a_{i}-n_{i}$ where $n_{i}$ is the largest integer such that $p a_{i} \geqq n_{i}$; then $\sum_{i=0}^{m} b_{i} A_{i}$ $\in \log I,\left|b_{i}\right|<1$ for $i=0,1, \cdots, m$, and $\left|\sum_{i=0}^{m} b_{i} A_{i}\right| \leqq \sum_{i=0}^{m}\left|A_{i}\right|$. Therefore, for each positive integer $k$, there is a matrix $B_{k}$ and a sequence $\left\{b_{k i}\right\}_{i=0}^{m}$ of numbers such that $B_{k}=\sum_{i=0}^{m} b_{k i} A_{i}$ is a logarithm of $I,\left|B_{k}\right| \leqq \sum_{i=0}^{m}\left|A_{i}\right|, b_{k 0}$ is an irrational number and $\left|b_{k+1,0}\right|$ $<\left|b_{k 0}\right|$.

Since $\left\{B_{k}\right\}_{\boldsymbol{k}=1}^{\infty}$ is a bounded sequence, it has a convergent subsequence and there exist two integers $r$ and $t$ such that $\left|B_{r}-B_{t}\right|<1$. From Theorem 4, it follows that $B_{r}-B_{t}=0$. However, $B_{r}-B_{t}$ $=\sum_{i=0}^{m}\left(b_{r i}-b_{t i}\right) A_{i} \neq 0$ because $A_{0}, A_{1}, \cdots, A_{m}$ are linearly independent and $b_{r 0} \neq b_{t 0}$.

Lemma 2. If $m$ and a are positive integers such that a does not divide $m$, then there are integers $p$ and $q$ and $a$ reduced fraction $c / b$ such that $p / m+q / a=c / b$ and $b>m$ (Corollary to $[2$, Theorem 25, p. 21]).

LemMa 3. If $S$ is a commutative subset of $\log I$ which is closed with respect to addition and subtraction, $a / b$ is a reduced fraction, $A \in S$, $(a / b) A+B \in S$ and $p$ is an integer, then there is an integer $q$ such that $(p / b) A+q B \in S$.

THEOREM 11. Suppose $S$ is a commutative subset of $\log I$ which is closed with respect to addition and subtraction. Conclusion. There exist an integer $m \leqq 2 n^{2}$ and linearly independent elements $B_{1}, B_{2}, \cdots, B_{m}$ of $S$ such that if $B \in \boldsymbol{S}$ then there exist integers $b_{1}, b_{2}, \cdots, b_{m}$ such that $B=\sum_{i=1}^{m} b_{i} B_{i}$.

Proof. Since $S$ is a subset of the linear vector space of $n \times n$ matrices with complex elements, then there exist, over the field of real numbers, $m$ linearly independent elements $A_{1}, A_{2}, \cdots, A_{m}$ of $S$ which span $S$ and $m \leqq 2 n^{2}$. By Theorem 15 if $\left\{p_{i}\right\}_{i=1}^{k}$ is a sequence of real numbers such that $\sum_{i=1}^{k} p_{i} A_{i} \in S$, then each of $p_{1}, p_{2}, \cdots, p_{k}$ is a rational number. 
For each integer $t=1,2, \cdots, m$, let $K_{t}$ denote the set of positive integers such that $k \in K_{t}$ if and only if there is a sequence $\left\{a_{i} / b_{i}\right\}_{i=t}^{m}$ of reduced fractions such that $\sum_{i=t}^{m}\left(a_{i} / b_{i}\right) A_{i} \in S,\left|a_{i} / b_{i}\right|<1$ for $i=t, t+1, \cdots, m$ and $k=b_{t}>0 . K_{t}$ is not an infinite set. If this is false, there exists a set $\left\{M_{j}\right\}_{j=1}^{\infty} \subset S$ such that $\left|M_{j}\right|<\sum_{i=1}^{m}\left|A_{i}\right|$ for $j=1,2,3, \cdots$, and $M_{k} \neq M_{j}$ if $k \neq j$; therefore, there exist two positive integers $p$ and $q$ such that $M_{p} \neq M_{q},\left|M_{p}-M_{q}\right|<1$ and from Theorem $9 M_{p}=M_{q}$. Hence, if $K_{t}$ is nonempty, it is a finite set and has a largest positive integer $m_{t}$.

If $1 \leqq t \leqq m$ and $\sum_{i=t}^{m}\left(a_{i} / b_{i}\right) A_{i} \in S$ and $a_{t} / b_{t}$ is a reduced fraction, then $b_{t}$ divides $m_{t}$. Suppose false; then, if $b_{t}>0$, by Lemma 2 , there exist in tegers $p$ and $q$ and a reduced fraction $k / b$ such that $b>m_{t}$ and $p / m_{t}+q / b_{t}=k / b$. By Lemma 3 , there are matrices $C$ and $D$ of the form $\sum_{i=t+1}^{m}\left(a_{i} / b_{i}\right) A_{i}$ such that $\left(p / m_{t}\right) A_{t}+C \in S$ and $\left(q / b_{t}\right) A_{t}$ $+D \in S$; therefore,

$$
(k / b) A_{t}+C+D=\left(\left(p / m_{t}\right) A_{t}+C\right)+\left(\left(q / b_{t}\right) A_{t}+D\right) \in S ;
$$

hence, $b \in K_{t}$ and $b \leqq m_{t}$, which contradicts $b>m_{t}$. Similarly, if $b_{t}<0$, then $a_{t} / b_{t}=-a_{t} /-b_{t}$ and $-b_{t}$ divides $m_{t}$.

If $0 \leqq t \leqq m, K_{t}$ is nonempty and $m_{t}$ is the largest integer in $K_{t}$, then by Lemma 3 there is a matrix $B_{t}$ and a sequence $\left\{c_{t i}\right\}_{i=t+1}^{m}$ of reduced fractions such that $B_{t}=\left(1 / m_{t}\right) A_{t}+\sum_{i=t+1}^{m} c_{t i} A_{i}$ and $B_{t} \in S$. These matrices $B_{1}, B_{2}, \cdots, B_{m}$ are linearly independent and will satisfy the conclusion of the theorem. If $B \in S$, there are reduced fractions $a_{1} / b_{1}, k_{12}, k_{13}, \cdots, k_{1 m}$ such that $B=\left(a_{1} / b_{1}\right) A_{1}+\sum_{i=2}^{m} k_{1 i} A_{i}$ belongs to $S$; also, there exists an integer $x_{1}$ such that $m_{1}=x_{1} b_{1}$. Since $B_{1}=1 / m_{1} A_{1}$ $+\sum_{i=2}^{m} c_{1 i} A_{i}$, then

$$
\begin{aligned}
B & =B-a_{1} x_{1}\left(B_{1}-B_{1}\right) \\
& =\left(a_{1} / b_{1}\right) A_{1}+\sum_{i=2}^{m} k_{1 i} A_{i}-a_{1} x_{1}\left(\left(1 / m_{1}\right) A_{1}+\sum_{i=2}^{m} c_{1 i} A_{i}\right)+a_{1} x_{1} B_{1} \\
& =a_{1}\left(1 / b_{1}-x_{1} / m_{1}\right) A_{1}+\sum_{i=2}^{m}\left(k_{1 i}-a_{1} x_{1} c_{1 i}\right) A_{i}+a_{1} x_{1} B_{1} \\
& =0+\sum_{i=2}^{m} k_{2 i} A_{i}+a_{1} x_{1} B_{1}, \quad \text { where } k_{2 i}=k_{1 i}-a_{1} x_{1} c_{1 i} .
\end{aligned}
$$

Similarly, there are integers $a_{2}$ and $x_{2}$ and a sequence $\left\{k_{3 i}\right\}_{i=3}^{m}$ of rational numbers such that

$$
\sum_{i=2}^{m} k_{2 i} A_{i}=a_{2} x_{2} B_{2}+\sum_{i=3}^{m} k_{3 i} A_{i} .
$$


By continuing this procedure with $B_{3}, \cdots, B_{m}$, we define sequences $\left\{a_{i}\right\}_{i=1}^{m}$ and $\left\{x_{i}\right\}_{i=1}^{m}$ of integers such that $B=\sum_{i=1}^{m} a_{i} x_{i} B_{i}$.

THEOREM 12. If $S$ is a commutative subset of $\log A$, then there exist an integer $m \leqq 2 n^{2}$ and a sequence $\left\{C_{i}\right\}_{i=1}^{m}$ of linearly independent commutative elements of $\log I$ such that if $P$ and $Q$ belong to $S$, then there exists a sequence $\left\{a_{i}\right\}_{i=1}^{m}$ of integers such that $P=Q+\sum_{i=1}^{m} a_{i} C_{i}$. Fur thermore, for $i=1,2, \cdots, m, C_{i}$ commutes with each element of $S$.

Proof. If $P$ and $Q$ belong to $S$, then $P-Q \in \log I$. Let $R$ be the subset of $\log I$ such that $B \in R$ if and only if there exist sequences $\left\{A_{i}\right\}_{i=1}^{k}$ and $\left\{B_{i}\right\}_{i=1}^{k}$ of elements of $S$ and a sequence $\left\{a_{i}\right\}_{i=1}^{k}$ of integers such that $B=\sum_{i=1}^{k} a_{i}\left(A_{i}-B_{i}\right)$. Theorem 11 holds and assures the existence of a sequence $\left\{C_{i}\right\}_{i=1}^{m}$ of linearly independent elements of $R$ such that if $P$ and $Q$ belong to $S$, then $P-Q$ belongs to $R$ and there exists a sequence $\left\{a_{i}\right\}_{i=1}^{m}$ of integers such that $P-Q$ $=\sum_{i=1}^{m} a_{i} C_{i}$. Furthermore, all the elements of $R$ and $S$ commute.

\section{BibliograPhy}

1. Richard Bellman, Introduction to matrix analysis, McGraw-Hill, New York, 1960.

2. G. H. Hardy and E. M. Wright, An introduction to the theory of numbers, 4 th ed., Oxford Univ. Press, London, 1965.

3. Einar Hille and Ralph S. Phillips, Functional analysis and semi-groups, rev. ed., Amer. Math. Soc. Colloq. Publ., Vol. 31, Amer. Math. Soc., Providence, R. I., 1957.

4. Von Mitio Nagumo, Einige analytische Untersuchungen in linearen metrischen Ringen, Japan. J. Math. 13 (1936), 61-80.

Southwest Texas State College 